\title{
Severe Demyelinating Neuropathy in an Advanced Melanoma Patient Treated with Nivolumab plus Ipilimumab Combined Therapy
}

\author{
Yumi Kambayashia ${ }^{a}$ Taku Fujimura $^{a}$ Hiroshi Kuroda $^{b}$ Atsushi Otsukac \\ Hiroyuki Irie $^{c}$ Setsuya Aiba ${ }^{a}$ \\ aDepartment of Dermatology, Tohoku University Graduate School of Medicine, Sendai, Japan; \\ bepartment of Neurology, Tohoku University Graduate School of Medicine, Sendai, Japan; \\ 'Department of Dermatology, Kyoto University Graduate School of Medicine, Kyoto, Japan
}

\section{Keywords}

Demyelinating peripheral neuropathy · Nivolumab plus ipilimumab combined therapy . Advanced melanoma $\cdot$ Immune-related adverse event $\cdot$ High-dose methylprednisolone monotherapy

\section{Abstract}

Immune checkpoint inhibitors (ICls) significantly prolong survival in patients with metastatic melanoma but can lead to serious immune-related adverse events. In this report, we described a case of atypical neuropathy caused by nivolumab plus ipilimumab combination therapy before primary tumor resection. In our case, not only demyelinating neuropathy, but also muscle weakness and unilateral facial nerve palsy developed and manifested as severe and diverse symptoms. Moreover, unlike spontaneously developing demyelinating peripheral neuropathy, the present case suggested the therapeutic effects of high-dose methylprednisolone monotherapy for the treatment of ICls-induced immune-related demyelinating peripheral neuropathy.

\section{Introduction}

Immune checkpoint inhibitors (ICIs), such as anti-PD1 antibodies and anti-CTLA4 antibodies, significantly prolong survival in patients with metastatic melanoma. However, ICIs can also lead to serious immune-related adverse events, especially with the administration of nivolumab plus ipilimumab (N + I) combined therapy [1]. In this report, we describe the case of a patient with advanced melanoma treated with $\mathrm{N}+\mathrm{I}$ combined therapy, which led to the 

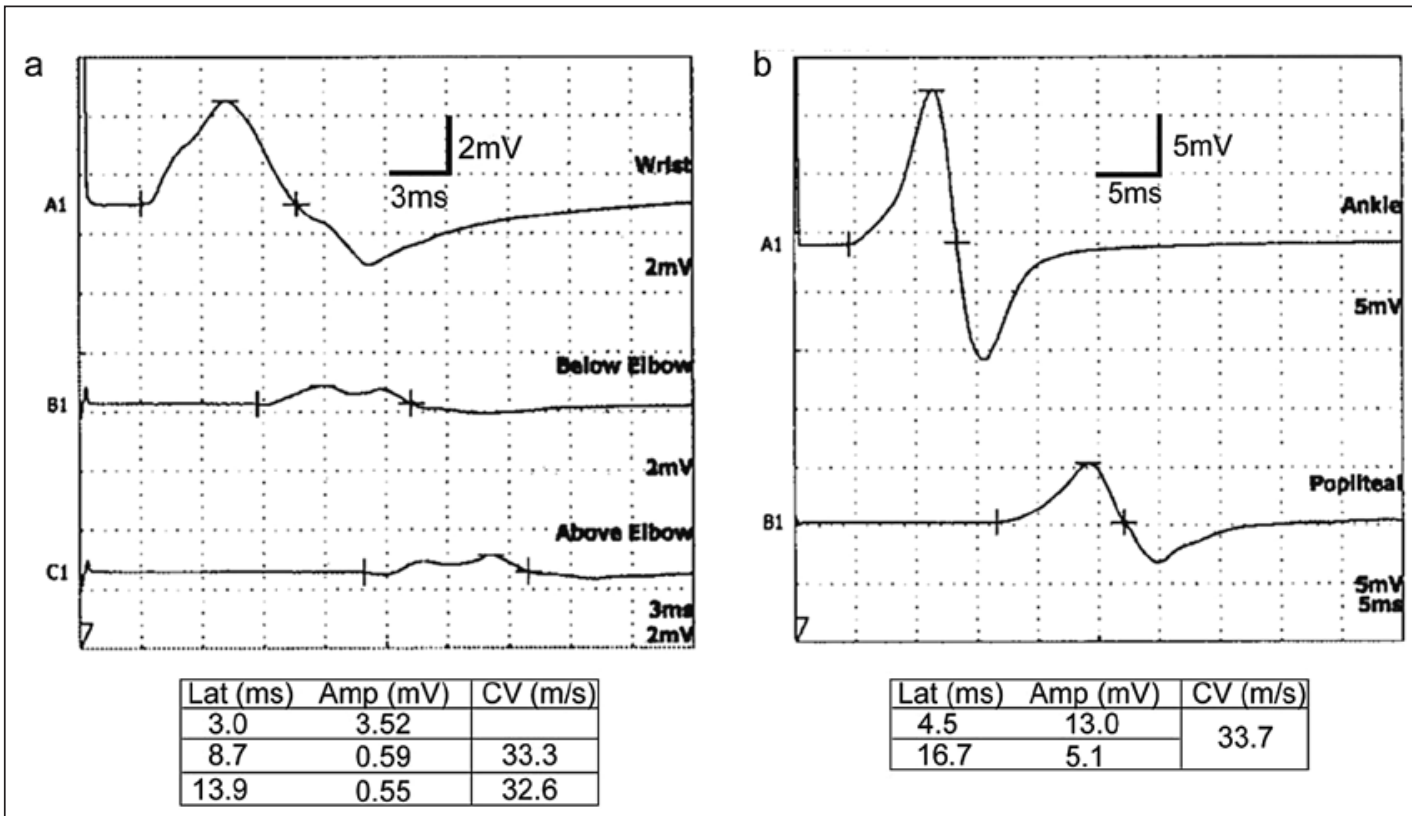

Fig. 1. Nerve conduction studies in the present case. a Motor conduction study of the right ulnar nerve shows prolonged latency in proximal stimulations, conduction block between the wrist and elbow, and decreased conduction velocity between the wrist and elbow and across the elbow. $\mathbf{b}$ Motor conduction study of the right posterior tibial nerve shows prolonged latency in proximal stimulation, conduction block between the ankle and popliteal fossa, and decreased conduction velocity between the ankle and popliteal fossa.

development of severe demyelinating peripheral neuropathy. Interestingly, this patient showed HLA-DQB1 polymorphisms (DQB1*040101 and *060401) that might have been associated with the immune-mediated neuropathy [2].

\section{Case Presentation}

We had previously reported on a 64-year-old Japanese man in Frontiers in Medicine in 2019 [3]. Since his melanoma had metastasized to multiple organs (skin, muscle, lymph nodes, oropharynx, lung, peritoneum, spleen, and bone), we administered ipilimumab ( $3 \mathrm{mg}$ / $\mathrm{kg})$ plus nivolumab $(80 \mathrm{mg} /$ body) $(\mathrm{N}+\mathrm{I})$ combined therapy prior to tumor resection. Ten days after the initial treatment, small areas of erythema and papules developed over approximately $30 \%$ of the body (grade 3), although these improved with oral prednisolone (PSL) at $30 \mathrm{mg} /$ day. The primary tumor was resected 18 days after the first administration of ICIs. Four cycles of the combination therapy were completed over 10 weeks without any severe adverse events other than the rash. Ten days after the fourth administration of $\mathrm{N}+\mathrm{I}$ combined therapy, he complained of strong pain in the right eye, numbness in the right face, and blurred vision in both eyes. We consulted an ophthalmologist, and bilateral uveitis (grade 2) was diagnosed.

In addition to bilateral uveitis, he complained about hypesthesia and pain in the territory of the right maxillary nerve and dysesthesia in all 4 limbs (grade 2-3) at the same moment. We consulted neurologists, who diagnosed drug-induced polyneuropathy. The patient was intravenously administered methylprednisolone (mPSL) at a dose of $1 \mathrm{mg} / \mathrm{kg} /$ day. Brain 
Kambayashi et al.: Severe Demyelinating Neuropathy by N + I Combined Therapy

magnetic resonance imaging revealed enhancement on the right trigeminal nerve, which was considered to represent the source of right facial pain. Twelve weeks after the first administration of ICIs, he developed right peripheral facial nerve palsy, weakness in all the limbs (most prominently in the right upper limb), with diminished deep tendon reflexes in the lower limbs, and sensory impairment with dysesthesia and paresthesia in the distal limbs. Moreover, he became unable to stand and walk independently due to limb weakness with generalized areflexia 13 days after the onset of pain in the right eye. Nerve conduction studies for the right ulnar nerve and posterior tibial nerve revealed prolonged distal latency, conduction block, and decreased conduction velocity, suggesting demyelinating neuropathy (Fig. 1). We finally diagnosed immune-related demyelinating peripheral neuropathy and, therefore, increased the dose of mPSL to $2 \mathrm{mg} / \mathrm{kg} /$ day for 3 weeks. Motor and sensory symptoms subsequently showed gradual improvement.

After 3 weeks, we gradually tapered the dose of intravenous mPSL from 2 to $1 \mathrm{mg} / \mathrm{kg} /$ day, then switched to oral PSL at a dose of $60 \mathrm{mg}$ /day and tapered that by decreasing the dose by $5 \mathrm{mg}$ /day every other week. Muscle weakness ameliorated in parallel with the improvement of nerve conduction studies. At 87 days after the development of right eye pain, he was able to walk unassisted, but mild facial nerve palsy remained.

To further clarify the immunological background that might correlate with immunerelated demyelinating peripheral neuropathy, we performed the human leukocyte antigen (HLA) analysis, which revealed that this patient possessed HLA-DQB1 polymorphisms (DQB1*040101 and *060401).

\section{Discussion}

In this report, we have described a case of immune-related demyelinating peripheral neuropathy with cranial neuropathy caused by $\mathrm{N}+\mathrm{I}$ combined therapy for advanced melanoma, successfully treated with high-dose mPSL $(2 \mathrm{mg} / \mathrm{kg}$ /day). Neurotoxicity is a rare immune-related adverse event in patients who have been treated with ICIs [4]. Indeed, the frequency of neurotoxicity is $1 \%$ with anti-CTLA4 antibody monotherapy, $3 \%$ with anti-PD-1 antibody monotherapy, and $14 \%$ with $\mathrm{N}+\mathrm{I}$ combined therapy [4]. The onset period appears to be about 12 months after starting monotherapy, whereas symptoms manifest within the first 3 months in combination therapy [4]. Histological findings for neuropathy caused by ICIs include dense infiltration of lymphocytes around microvessels associated with nerves, suggesting that this neuropathy might be similar to autoimmune inflammatory neuropathy [5]. Since melanocytes and Schwann cells share several similar antigens, such as S100, treatment with ICIs against melanoma might lead to the development of autoimmune reactions against Schwann cells [6]. In addition, in the present case, we administered N + I combined therapy in a neoadjuvant setting, which could have strongly induced melanomareactive T-cell clones at the tumor site [7], leading to migration into peripheral blood and the development of widespread autoimmune-like neuropathy. Indeed, various neurotoxicities caused by ICIs have been reported, including peripheral neuropathy, Guillain-Barré syndrome, cranial nerve palsy, demyelinating disease, myasthenia gravis syndrome, meningitis, encephalitis, central nervous system demyelination, optic neuritis, transverse myelitis, and ipsilateral mononeuritis, such as Bell's palsy [4]. For ICIs-related neuropathies, such as those mentioned above, several treatments have been reported, generally starting with oral or intravenous injection of PSL at $0.5-1.0 \mathrm{mg} / \mathrm{kg}$ bodyweight, mPSL at $1-2 \mathrm{mg} / \mathrm{kg}$, and then consideration of intravenous immunoglobulin and plasmapheresis as additional therapies for steroid-resistant patients [4]. Approximately one-third of patients who develop immunerelated neurotoxicity remain with some degree of residual dysfunction [4]. On the other hand, 
patients who developed neurotoxicity from ICIs have shown longer progression-free and overall survival than those who did not.

In this report, we described an atypical neuropathy caused by $\mathrm{N}+\mathrm{I}$ combination therapy before primary tumor resection. Although the clinical type of demyelinating neuropathy with weakness of the whole body including the face was Guillain-Barré syndrome-like in our present case, the response to steroids was similar to chronic demyelinating neuropathy, which was represented by chronic inflammatory demyelinating polyneuropathy. Ten months after symptom onset, facial nerve palsy remains despite continued administration of PSL at $8 \mathrm{mg} /$ day. Unlike spontaneously developing demyelinating peripheral neuropathy, the present case suggests that high-dose mPSL monotherapy, without intravenous immunoglobulin, is useful for treating ICIs-induced immune-related demyelinating peripheral neuropathy.

\section{Statement of Ethics}

The patient has given his written informed consent to the publication of his case.

\section{Disclosure Statement}

The authors have no conflicting interests to declare.

\section{Funding Sources}

There were no funding sources.

\section{Author Contributions}

Y. Kambayashi designed the research study. Y. Kambayashi, T. Fujimura, and H. Kuroda treated the patient and acquired the clinical data. A. Otsuka and H. Irie performed HLA typing. Y. Kambayashi, T. Fujimura, and H. Kuroda wrote the manuscript. T. Fujimura and S. Aiba supervised the study.

\section{References}

1 Wolchok JD, Chiarion-Sileni V, Gonzalez R, Rutkowski P, Grob JJ, Cowey CL, et al. Overall survival with combined nivolumab and ipilimumab in advanced melanoma. N Engl J Med. 2017;377(14):1345-56.

2 Hayat S, Jahan I, Das A, Hassan Z, Howlader ZH, Mahmud I, et al. Human leukocyte antigen-DQB1 polymorphisms and haplotype patterns in Guillain-Barré syndrome. Ann Clin Transl Neurol. 2019;6(9):1849-57.

3 Fujimura T, Kambayashi Y, Sato Y, Tanita K, Amagai R, Hashimoto A, et al. Successful treatment of unresectable advanced melanoma by administration of nivolumab with ipilimumab before primary tumor resection. Front Med (Lausanne). 2019;6:140.

4 Spain L, Tippu Z, Larkin JM, Carr A, Turajlic S. How we treat neurological toxicity from immune checkpoint inhibitors. ESMO open. 2019;4(Suppl 4):e000540.

5 Spain L, Walls G, Julve M, O'Meara K, Schmid T, Kalaitzaki E, et al. Neurotoxicity from immune-checkpoint inhibition in the treatment of melanoma: A single centre experience and review of the literature. Ann Oncol. 2017; 28(2):377-85.

6 Liao B, Shroff S, Kamiya-Matsuoka C, Tummala S. Atypical neurological complications of ipilimumab therapy in patients with metastatic melanoma. Neuro-oncology. 2014;16(4):589-93.

7 Blank CU, Rozeman EA, Fanchi LF, Sikorska K, van de Wiel B, Kvistborg P, et al. Neoadjuvant versus adjuvant ipilimumab plus nivolumab in macroscopic stage iii melanoma. Nat Med. 2018;24(11):1655-61. 